\title{
Habilidades para Teletrabalho em Casa: Construção e Evidências de Validade da Escala
}

\author{
Gardenia da Silva Abbad ${ }^{1, *}$, Luciana Mourão ${ }^{2}$, Russencleyton Barros Costa ${ }^{3}$, \\ Lara Barros Martins ${ }^{4}$, Juliana Legentil ${ }^{5}$, Lisa Miranda ${ }^{6}$
}

\footnotetext{
${ }^{1}$ http://orcid.org/0000-0003-0807-3549 / Universidade de Brasília (UnB), Brasil

${ }^{2}$ http://orcid.org/0000-0002-8230-3763 / Universidade Salgado de Oliveira (Universo), Brasil; Universidade do Estado do Rio de Janeiro (UERJ), Brasil

3 http://orcid.org/0000-0002-4833-7783 / Universidade Salgado de Oliveira (Universo), Brasil; Instituto Nacional de Tecnologia (INT), Brasil

${ }^{4}$ http://orcid.org/0000-0002-3175-6324 / Universidad Loyola, Espanha

${ }^{5}$ http://orcid.org/0000-0002-9512-983X / Universidade de Brasília (UnB), Brasil

${ }^{6}$ http://orcid.org/0000-0002-7029-4994 / Universidade de Brasília (UnB), Brasil
}

Resumo

Muitos trabalhadores migraram para o teletrabalho durante a pandemia de COVID-19. A realização das tarefas integralmente em casa trouxe desafios, principalmente àqueles que não possuíam experiência anterior com a modalidade, como a conciliação trabalho-família e o uso de tecnologias digitais. Este estudo relata o desenvolvimento e as evidências de validade psicométrica da Escala de Habilidades para o Teletrabalho em Casa (EHTC). A pesquisa foi aplicada a 7.608 servidores de diferentes órgãos públicos brasileiros. Realizaram-se análises estatísticas descritivas, fatoriais exploratórias e confirmatórias e de confiabilidade. O instrumento possui uma estrutura empírica bifatorial que avalia habilidades para o balanceamento entre trabalho e outras atividades (soft skills) e para utilização de recursos digitais nas interações de trabalho (hard skills). A EHTC pode ser usada para identificar a adaptação e o preparo para o teletrabalho em casa e investigar associações entre essas habilidades e outros indicadores.

Palavras-chave: trabalho remoto, soft skills, hard skills.

\section{Skills for Working from Home: Construction and Validation of the Scale}

\section{Habilidades para el Teletrabajo en Casa: Construcción y Evidencias de Validad de la Escala}

\section{Abstract}

Many workers migrated to teleworking during the COVID-19 pandemic. Performing work tasks entirely at home brought challenges, especially for those who had no previous experience with the modality, such as work-family conciliation and the use of digital technologies. This study reports the development and evidence of psychometric validity of the Skills for Working from Home scale (SWHS). The survey was applied to 7,608 government employees from Brazilian public agencies. Descriptive statistical analyses, and exploratory, confirmatory, and reliability factor analyses were performed. The instrument has a bifactorial empirical structure that assesses skills for balancing work and other activities (soft skills) and skills for using digital communication resources in work interactions (hard skills). The SWHS can be used to identify the adaptation and preparation for working from home, as well as to investigate possible associations between these skills and other indicators.
Resumen

Muchos trabajadores migraron para el teletrabajo durante la pandemia de COVID-19. Realizar las tareas íntegralmente en casa trajo desafíos, especialmente para quienes no tenían experiencia previa con la modalidad, como la conciliación trabajo-familia y el uso de tecnologías digitales. Este estudio reporta el desarrollo y las evidencias de validad psicométrica de la Escala de Habilidades para el Teletrabajo en Casa (EHTC). La investigación se aplicó a 7.608 funcionarios de organos públicos brasileños. Se realizaron análisis estadísticos descriptivos, factoriales exploratorios, confirmatorios y de confiabilidad. El instrumento posee una estructura empírica bifactorial que evalúa habilidades para equilibrar el trabajo y otras actividades (soft skills) y para el uso de recursos digitales en las interacciones laborales (hard skills). La EHTC puede ser utilizada para identificar la adaptación y preparación para el teletrabajo en casa e investigar posibles asociaciones entre estas habilidades y otros indicadores. 
A pandemia da COVID-19 provocou uma grande transformação no mundo do trabalho e no cotidiano das pessoas em muitos países. Uma das repercussões para os trabalhadores e as organizações dos mais diversos segmentos foi a migração repentina para o trabalho remoto, em tempo integral e realizado em casa, sem preparo prévio (Kniffin et al., 2021). Além da adaptação brusca às medidas de isolamento preconizadas pela crise sanitária mundial instaurada em março de 2020, o teletrabalho emergencial introduziu novos desafios e demandas de trabalho, requerendo o desenvolvimento de habilidades para equilibrar tarefas laborais e domésticas, bem como utilizar tecnologias digitais requeridas pela modalidade (Losekann \& Mourão, 2020). Nesse sentido, este trabalho objetivou desenvolver e verificar evidências de validade psicométrica de um instrumento para mensurar habilidades de adaptação ao trabalho remoto em casa.

Antes da pandemia, o trabalho remoto não era amplamente utilizado pelos trabalhadores. Nos EUA, em 2017, o trabalho remoto doméstico (Work From Home - WFH) era praticado por $2,9 \%$ da força de trabalho; na Europa, em 2015, por apenas $2 \%$ dos trabalhadores (Eurofound, 2015). No Brasil, em 2018, um total de 3,8 milhões de pessoas trabalhavam em suas residências, sem especificar a natureza das atividades realizadas (Instituto Brasileiro de Geografia e Estatística, 2018). Além disso, o teletrabalho vinha sendo praticado por adesão voluntária, em geral por trabalhadores em cargos executivos e gerenciais ou profissionais do ramo tecnológico (Losekann \& Mourão, 2020; Wang, Liu, Qian, \& Parker, 2021).

Nos últimos anos, observou-se que o número de pessoas em trabalho remoto cresceu em especial nos países mais desenvolvidos, como o Reino Unido e os EUA, que já possuíam maior potencial especificamente para o trabalho em casa. O Brasil ocupava, antes da pandemia, a $47^{a}$ posição em potencial de realização do teletrabalho em casa, em uma lista de 86 países, conforme Dingel e Neiman (2020). Segundo estimativas desses autores, o Brasil possuía um potencial de $25,7 \%$ de pessoas que podiam realizar seu trabalho inteiramente em casa, contra a estimativa de $22,7 \%$ de pessoas (20,8 milhões) calculada a partir da Pesquisa Nacional por Amostra de Domićlios (PNAD), do Instituto de Pesquisa Econômica Aplicada (Ipea) (Goes, Ramos, \& Ferreira, 2020).

Essas estimativas variaram de acordo com as unidades da federação brasileira e com as ocupações. O Distrito Federal apresentou o maior percentual de teletrabalho em casa do país $(31,6 \%)$, atingindo em torno de 450 mil pessoas, e o Piauí, o menor percentual (15,6\%), cerca de 192 mil pessoas. As ocupações com maior potencial de realização do trabalho em casa foram: profissionais das ciências e intelectuais (65\%), diretores e gerentes (61\%), profissionais de nível médio $(30 \%)$ e trabalhadores de apoio administrativo (12\%) (Goes et al., 2020).

A renda per capita dos estados está positivamente correlacionada ao potencial de teletrabalho em casa, colocando Distrito Federal, São Paulo e Rio de Janeiro com os maiores percentuais de pessoas trabalhando remotamente e com potencial de teletrabalho. A quantidade de pessoas em trabalho remoto residencial cresceu de 3,8 milhões, em 2018, para 8,7 milhões, em 2020, como resultado das medidas de distanciamento social para combater a pandemia. Esse aumento indica que milhares de pessoas não possuíam experiência anterior com o trabalho remoto, mediado integralmente por tecnologias digitais e realizado em casa. Em maio de 2020, no Brasil, havia 13,3\% das pessoas ocupadas exercendo suas atividades remotamente (Goes et al., 2020).

Com relação à regulação do trabalho remoto em órgãos públicos, normativas foram propostas para viabilizar a continuidade das atividades administrativas em meio à pandemia, com teletrabalho em caráter excepcional e provisório no Executivo Distrital
(Decreto $\mathrm{n}^{\circ} 40.546$, de 20 de março de 2020, revogado e substituído pelo Decreto $n^{\circ} 41.841$, de 26 de fevereiro de 2021) e no Executivo Federal (Instrução Normativa no 19, de 12 de março de 2020).

Os substanciais cortes de gastos, considerando apenas os primeiros meses da pandemia e a migração para o trabalho remoto em larga escala - $\mathrm{R} \$ 36,4$ milhões para o governo distrital e cerca de $\mathrm{R} \$ 1$ bilhão entre servidores públicos (Agência Brasília, 2020; Ministério da Economia, 2020) -, aliados aos indicadores de manutenção da produtividade levaram à decisão de manter o teletrabalho de forma definitiva. A publicação de uma nova instrução normativa sobre a implementação de programa de gestão em 2020, mais simples e flexível, possibilita que diversas organizações públicas possam manter servidores trabalhando remotamente após a pandemia da COVID-19, com foco em resultados (Instrução Normativa $n^{\circ}$ 65, de 30 de julho de 2020).

Entre as consequências diretas da adoção do WFH, estão mudanças nas práticas de trabalho, exigindo grande capacidade de adaptação das pessoas ao novo contexto e à modalidade. Os trabalhadores precisaram, sem preparação prévia, conciliar trabalho com outras atividades (domésticas, acompanhamento dos filhos em ensino remoto etc.), estabelecer limites entre as fronteiras de trabalho e não trabalho, atuar em equipes virtuais e sem contato face a face com colegas de trabalho, realizar todas as atividades laborais mediadas por tecnologias digitais, comunicar-se de forma síncrona e assíncrona e vivenciar perda de suporte social e das redes de apoio, em especial nos casos de trabalhadores com filhos dispensados das escolas (Kniffin et al., 2021; Wang et al., 2021).

As mudanças, principalmente, nas configurações dos contextos físico e social de trabalho exigiram uma adaptação rápida desses trabalhadores para equilibrar trabalho remoto e outras atividades, manter a produtividade e assegurar-se de estabelecer uma jornada saudável de trabalho. Em decorrência dos limites mais tênues entre trabalho e não trabalho, a preocupação em realizar intervalos para descanso e recuperação de energia exigiu um esforço adicional (Sandall \& Mourão, 2020) a fim de evitar o estresse e a exaustão - considerando, inclusive, que as medidas restritivas reduziram as chances de lazer e descanso. No mesmo sentido, as habilidades para utilização de recursos digitais de comunicação nas interações de trabalho, apesar de viabilizarem e facilitarem o trabalho remoto, ao serem utilizadas intensivamente, podem ocasionar interferência na relação trabalho-família e tornar a comunicação pouco efetiva (Wang et al., 2021). Por esses motivos, possuir habilidades requeridas pelo teletrabalho compulsório no contexto da pandemia, e sentir-se capaz de expressá-las, tornou-se um diferencial de trabalhadores que passaram a desempenhar suas funções remotamente em casa.

Assim, propor uma medida inédita com a finalidade de avaliar as habilidades de adaptação ao WFH, aplicada a servidores públicos, atende a um enorme potencial de atividades elegíveis para o teletrabalho, produzindo conhecimentos de relevância social acerca das experiências de trabalhadores expostos a novas condições e demandas de trabalho durante o enfrentamento da pandemia da COVID-19. Tal ferramenta de diagnóstico permite a autoavaliação do nível de preparo quanto ao domínio de habilidades para o teletrabalho em casa e pode ser utilizada em outras amostras e contextos antes da implementação da modalidade remota de trabalho. Ao avaliar a percepção de trabalhadores sobre suas próprias habilidades para atuar no teletrabalho, intervenções podem ser propostas com a finalidade de suprir lacunas identificadas, como programas integrados de treinamento e de promoção ao bem-estar e à qualidade de vida no trabalho (Abbad \& Legentil, 2020). 


\section{Habilidades para o Teletrabalho em Casa}

Algumas demandas de aprendizagem dizem respeito a habilidades, comportamentais e técnicas, necessárias à realização das tarefas integralmente a partir de casa (soft skills) e com a mediação de tecnologias digitais (hard skills). O primeiro conjunto de habilidades refere-se àquelas intra e interpessoais, relativas às formas de se relacionar e interagir com as pessoas (Gondim, Morais, \& Brantes, 2014). No caso do WFH, compreende tanto as relações e interações estabelecidas, a distância, com colegas de trabalho (pares e chefias) como as experienciadas, presencialmente, com os que convivem no lar (cônjuge, filhos etc.). Tais competências socioemocionais são aplicadas visando à busca por equilíbrio entre as esferas trabalho-família no que tange às atividades (laborais vs. domésticas), ao tempo dedicado ao cumprimento de metas de trabalho e ao descanso, e às relações e à comunicação estabelecidas em ambos os domínios. Para enfrentar essa situação em que trabalho e família disputam o mesmo espaço, ao mesmo tempo, os trabalhadores certamente passaram por um processo de adaptação e de desenvolvimento de competências que viabilizasse preservar os vínculos familiares e alcançar objetivos e resultados no trabalho (Martins, Aguiar, \& Bastos, 2020).

Além de equilibrar essas responsabilidades, teletrabalhadores devem demonstrar habilidades relacionadas à autonomia, autodisciplina, automotivação e autogerenciamento, que são capacidades autorregulatórias que auxiliam na manutenção de atenção e concentração nas atividades em andamento. A autodisciplina, por exemplo, foi indicada como uma característica individual diferencial para se beneficiar do teletrabalho (Wang et al., 2021). Desses profissionais serão também demandadas cotas mais altas para tomar decisões sobre a organização e o planejamento do trabalho, e, consequentemente, cumprir metas; administrar o tempo dedicado às tarefas; estabelecer intervalos (limites entre trabalho e não-trabalho); e desenvolver habilidades de comunicação (trabalhador com membros da família e colegas de trabalho).

Lacunas na expressão de tais habilidades já eram relatadas por teletrabalhadores, mas foram potencializadas durante a pandemia, principalmente para aqueles que nunca haviam sido expostos ao trabalho remoto, e que, repentinamente, tiveram que aprender a lidar com condições atípicas de trabalho no ambiente doméstico, algumas vezes associadas à procrastinação e à solidão (Wang et al., 2021). Adicionalmente, muitos trabalhadores não possuíam em casa um ambiente adequado (equipamentos, mobiliário, local isolado etc.) ao teletrabalho (Abbad \& Legentil, 2020).

Além disso, muitos trabalhadores tiveram que aprender habilidades técnicas, que dizem respeito ao domínio dos recursos tecnológicos e digitais (e.g., celulares, sistemas operacionais, aplicativos de mensagens e plataformas para videoconferências) demandados pela modalidade on-line, também necessárias para a execução a contento das atividades laborais. Essas habilidades, classificadas como hard skills, são instrumentais, e a necessidade de capacitação nesse tipo habilidade costuma ser mais facilmente detectável e treinável, além de receber mais suporte social e gerencial (Laker \& Powell, 2011).

No entanto, o fato de ser mais fácil treinar tais habilidades não indica que a comunicação mediada por tecnologias digitais não tenha problemas. Pelo contrário, a literatura de teletrabalho aponta que essa comunicação apresenta desvantagens quando comparada à interação face a face (Raghuram, Hill, Gibbs, \& Maruping, 2019). A demanda por uma comunicação eficaz no trabalho remoto é um elemento essencial para experiências bem-sucedidas nessa modalidade, nesse sentido, é fundamental considerar tais habilidades no contexto do teletrabalho (Wang et al., 2021).

O domínio de um conjunto de habilidades variadas, que combinam soft e hard skills, pode funcionar como propulsores de desempenho e outros resultados relevantes, como bem-estar no trabalho. As habilidades técnicas são importantes, pois facilitam a execução de tarefas, mas as socioemocionais provavelmente assumirão um lugar de destaque para fazer frente aos desafios impostos pelo WFH. As soft skills - também chamadas de competências sociais ou habilidades sociais - estão intimamente relacionadas à empregabilidade, sendo bastante demandadas pelo mercado de trabalho (Cinque, 2016). De acordo com Charoensap-Kelly, Broussard, Lindsly e Troy (2015), o termo soft skills vem sendo cada vez mais usado, estando o conceito associado à inteligência emocional, bem como a habilidades intra e interpessoais (socioemocionais) consideradas essenciais para o desenvolvimento pessoal e o profissional. Assim, as autoras distinguem os dois termos apontando que as hard skills estariam associadas ao know-how técnico tangível, enquanto as soft skills seriam habilidades mais abstratas, caracterizadas como qualidades pessoais ou interpessoais necessárias para adquirir um trabalho ou para se destacar nele.

Portanto, identificar e mensurar as demandas de aprendizagem tanto de hard skills como de soft skills contribui para decisões sobre programas de treinamento específicos para a habilidade a ser desenvolvida (Laker \& Powell, 2011), com vistas a instrumentalizar e oferecer aos trabalhadores remotos oportunidades de aprendizagem, capazes de sanar suas principais lacunas, preparando-os para uma atuação mais eficaz e saudável nesse contexto (Wang et al., 2021).

Nesta pesquisa, foram utilizados, como aportes teóricos à concepção dos itens da escala proposta, referenciais e modelos teóricos de Desenho do Trabalho (Morgeson \& Humphrey, 2006; Parker, 2014), Teoria de Demandas-Recursos do Trabalho (JD-R) (Bakker \& Demerouti, 2017) e Teoria de Conservação de Recursos (Hobfoll, 2001), pois essas teorias têm sido adotadas em estudos sobre o $W F H$, antes e durante a pandemia de COVID-19, em pesquisas sobre produtividade, riscos de exaustão, estresse e burnout. Essas teorias incluem características do ambiente de trabalho e dos trabalhadores que exercem influência sobre a produtividade, o bem-estar e outras importantes vivências psicológicas dos trabalhadores.

\section{Modelo de Desenho do Trabalho}

Com base nos modelos teóricos de Morgeson e Humphrey (2006) e Parker (2014), estudos como o de Wang et al. (2021) foram realizados para mapear, qualitativamente e quantitativamente, os principais desafios enfrentados por trabalhadores chineses no WFH. Entre as características-chave do trabalho virtual apontadas, estão o suporte social, a autonomia no trabalho, o monitoramento e a sobrecarga de trabalho - os dois primeiros funcionariam como recursos e os dois últimos, como demandas de trabalho.

As características sociais do trabalho mudaram bruscamente durante o período de pandemia ao passarem a exigir a mediação tecnológica para todas as interações síncronas e assíncronas entre colegas e superiores, dificultando o suporte social no trabalho. Os trabalhadores, ao desempenharem suas atividades em casa, precisam demonstrar mais autonomia para planejar e decidir sobre o modo de executar o trabalho e buscar por apoio sempre através de meios digitais. Tanto a autonomia como o suporte social foram indicados como preditores de vivências favoráveis de bem-estar e produtividade (Wang et al., 2021).

Assim, as experiências desafiadoras do trabalho remoto referidas tratam de interferência trabalho-ambiente doméstico, comunicação ineficaz, procrastinação e solidão (Wang et al., 2021). Evitar as distrações no ambiente doméstico, como interrupções de familiares, e as interferências negativas de um domínio sobre 
o outro (trabalho-família) demanda o desenvolvimento de uma habilidade de autogestão do trabalho (Sandall \& Mourão, 2020), o que requer o uso frequente de soft skills. Essas habilidades também serão fundamentais para aprimorar a comunicação entre os diversos atores envolvidos nesse contexto do WFH não voluntário (por exemplo, colegas, superiores e familiares) em busca de suporte social e de equilíbrio entre recursos e demandas de trabalho.

\section{Teoria de Demandas-Recursos do Trabalho (JD-R)}

De acordo com a Teoria $J D-R$, as características do trabalho são classificáveis como demandas ou recursos. Se aspectos físicos, psicológicos, organizacionais ou sociais do trabalho requerem esforço e acarretam custos (fisiológicos e psicológicos) ao trabalhador, são denominados de demandas; se esses mesmos aspectos reduzem as exigências e os custos do trabalhador, são considerados recursos. Esses aspectos são necessários ao alcance de objetivos de trabalho e estimulam o crescimento pessoal, o desenvolvimento e a aprendizagem (Demerouti, Bakker, Nachreiner, \& Schaufeli, 2001).

Nem sempre as demandas de trabalho são negativas, porém podem se tornar um obstáculo quando requerem esforço elevado e há oportunidades insuficientes de descanso e recuperação de energia. Enquanto as demandas, que exigem esforço e consomem recursos energéticos do indivíduo, são preditoras de esgotamento e problemas de saúde, os recursos, que apoiam processos de satisfação de necessidades humanas básicas, são preditores de satisfação, motivação e engajamento. Recursos pessoais, sociais e organizacionais podem atenuar os efeitos de demandas de trabalho sobre o bem-estar do trabalhador (Bakker \& Demerouti, 2017).

Há evidências de que a pandemia aumentou as demandas psicológicas e físicas de trabalho, e está submetendo os indivíduos ao risco de exaustão emocional e à solidão (Wang et al., 2021). O teletrabalho compulsório no contexto da COVID-19 demandou um esforço para o balanceamento entre trabalho e não trabalho, sobrecarga de tarefas e afazeres domésticos, busca de espaço para realizar tarefas sem interferências de outras pessoas, uso intensivo de tecnologias digitais para a realização de todas as atividades remotas, perda do suporte social face a face de colegas e familiares e demanda de reorganização da forma como o trabalho é realizado (Losekann \& Mourão, 2020; Sandall \& Mourão, 2020).

Quanto aos recursos organizacionais e sociais disponíveis para mitigar os efeitos dos fatores estressores, pode-se citar a flexibilidade na atribuição de tarefas e no monitoramento do alcance de metas de trabalho e a oferta de suporte tecnológico e social aos trabalhadores. Entre os recursos psicológicos, estão habilidades de adaptação e aprendizagem para atuação no contexto de trabalho remoto, autoeficácia e domínio de habilidades de uso de tecnologias digitais de comunicação e de busca de equilíbrio entre trabalho e outras atividades (Kniffin et al., 2021; Meseguer-de Pedro, Fernández-Valera, García-Izquierdo, \& Soler-Sánchez, 2021).

Por um lado, o teletrabalho compulsório pode atuar como um ambiente inibidor de estados psicológicos e resultados positivos, pois as condições de trabalho (materiais e psicossociais) podem ser inadequadas ao bom desempenho, atuando como demandas (Wang et al., 2021). Por outro lado, trabalhadores com habilidades variadas, que combinem soft e hard skills, podem obter melhores resultados no trabalho (Charoensap-Kelly et al., 2015).

\section{Teoria de Conservação de Recursos}

De acordo com a Teoria de Conservação de Recursos (Hobfoll, 2001), o conceito de recursos refere-se a características pessoais, condições, objetos ou energias que são valiosas por seus próprios atributos ou como meios para proteger os recursos disponíveis. Segundo essa abordagem, os indivíduos lutam para obter, manter, proteger e promover os recursos que valorizam. Dada a situação de maior estresse psicológico, como no caso do trabalho remoto no contexto restritivo da pandemia, esses recursos tornam-se ainda mais importantes.

O enfrentamento do isolamento social e do trabalho remoto implementado repentinamente, de acordo com esse tipo de abordagem, requer a mobilização de recursos pessoais e organizacionais que minimizem os efeitos negativos dessas experiências sobre o bem-estar e a saúde do trabalhador. Um exemplo de recurso é o capital psicológico, definido por Luthans, Youssef e Avolio (2007) como um fator de segunda ordem, que reúne otimismo, resiliência, esperança e autoeficácia. Esses recursos individuais caracterizam-se por atribuições otimistas sobre triunfos no presente e futuro; capacidade de se recuperar e de se fortalecer perante adversidades; visualização, perseverança e redirecionamento de objetivos para o alcance deles; e autoeficácia, i.e., confiança nas próprias capacidades de enfrentamento de desafios e dificuldades relacionados às tarefas.

A autoeficácia, especificamente, pode ser considerada um valioso recurso psicológico que pode motivar o trabalhador a adquirir novas aprendizagens (Charoensap-Kelly et al., 2015). A convicção que a pessoa possui sobre ser capaz de realizar uma tarefa específica parece desempenhar um importante papel em um contexto de pandemia, pois perceber a própria capacidade de expressar habilidades para lidar com condições e conflitos intra e interpessoais, bem como com as adaptações ao teletrabalho, pode gerar melhores resultados individuais e organizacionais.

Entretanto, o capital psicológico e seus componentes podem exaurir-se quando indivíduos submetidos a situações de alto estresse não conseguem conservar e obter mais recursos positivos para o enfrentamento dessas adversidades. Um estudo realizado na Espanha em dois momentos (o primeiro, no início do período de confinamento, em dezembro de 2019, e o segundo, em abril de 2020) ilustra muito bem o ciclo de perda de recursos psicológicos dos trabalhadores com a passagem do tempo. Os resultados mostraram que os recursos pessoais de enfrentamento da pandemia decaem com a passagem do tempo, pois houve uma redução nos níveis de autopercepção de saúde durante o confinamento obrigatório (Meseguer-de Pedro, Soler-Sánchez, Fernández-Valera, \& García-Izquierdo, 2017). O ciclo de perda de recursos pessoais pode levar ao aumento de estresse, exaustão e burnout. Por isso, é importante evitar que o teletrabalho compulsório seja mais uma demanda a prejudicar o bem-estar psicológico e a saúde do trabalhador em tempos de pandemia e identificar quais recursos pessoais (soft e hard skills) precisam ser desenvolvidos pelos trabalhadores para o enfrentamento saudável dessas transformações e novas demandas de trabalho.

\section{Método}

\section{Participantes}

Os participantes foram escolhidos de modo não aleatório e por conveniência. Participaram do estudo 7.608 servidores, sendo 5.966 distribuídos em 93 órgãos do Poder Executivo Distrital, 807 servidores vinculados a um dos órgãos do Ministério Público da União e 835 servidores do Poder Judiciário Federal. Nesses órgãos públicos, a autorização para o teletrabalho foi ampliada em caráter excepcional e provisório em razão da pandemia.

Após a coleta de dados, foi possível identificar que a amostra foi majoritariamente composta por participantes do sexo feminino $(59,3 \%)$, com faixas etárias entre 38 e 47 anos $(34,2 \%)$ e en- 
tre 28 e 37 anos (29,8\%), casados ou em união estável $(63,5 \%)$ e com pós-graduação stricto ou lato sensu $(60.5 \%)$, ensino superior $(32.9 \%)$ ou ensino médio (6.6\%). Em relação à experiência prévia com teletrabalho, $58.1 \%$ dos participantes declararam não ter nenhuma experiência. Daqueles $41.9 \%$ que tiveram experiência anterior com teletrabalho, $93.7 \%$ relatam que tiveram uma experiência positiva; e $6.3 \%$, uma experiência negativa.

\section{Instrumentos}

O instrumento foi construído a partir da análise da literatura sobre desafios, dificuldades, demandas, benefícios e barreiras associados ao teletrabalho em casa (home office, WFH) (Abbad et al., 2019; Aguilera, Lethiais, Rallet, \& Proulhac, 2016; Barros \& Silva, 2010; Biron \& van Veldhoven, 2016) e da avaliação do contexto de trabalho remoto compulsório, tal como instituído nos órgãos públicos previamente mencionados, nas primeiras semanas da decretação de regras de distanciamento social como medida de combate à pandemia.

Os itens da Escala de Habilidades para o Teletrabalho em Casa (EHTC), inspirados nos conceitos de autoeficácia, (auto) disciplina e processos autorregulatórios apontados em evidências recentes sobre o teletrabalho não voluntário (Kniffin et al., 2021; Meseguer-de-Pedro et al., 2021; Wang et al., 2021; Windeler, Chudoba, \& Sundrup, 2017), foram elaborados com vistas a aferir o quanto os teletrabalhadores se sentiam capazes ou habilitados a enfrentar com sucesso o período de adaptação ao trabalho remoto em casa e consideram as soft e hard skills.

As habilidades socioemocionais (soft skills) abrangem a busca de equilíbrio entre demandas de trabalho, família e afazeres domésticos; o cuidado com fatores causadores de sobrecarga e exaustão, como interrupções frequentes e falta de intervalos para descanso; e o planejamento e a gestão do tempo de trabalho e não trabalho e das interações com família, chefe e outras pessoas que possam interferir ou interromper a realização das tarefas. Dado o contexto de crise sanitária, essas habilidades podem ser consideradas recursos pessoais relevantes que possibilitam a manutenção da produtividade e propiciam um ambiente saudável de trabalho, ao reduzirem ou evitarem a sobrecarga, a exaustão e o estresse.

As habilidades instrumentais (hard skills) de uso de tecnologias digitais de comunicação passaram também a ser uma condição necessária à realização de todas as tarefas, ao alcance de metas de trabalho e ao estabelecimento de contatos com pares e chefia. Porém, nem sempre os profissionais possuem essas habilidades bem desenvolvidas. No serviço público, uma grande parcela de servidores não tinha experiência prévia de uso intensivo de tecnologias digitais de comunicação para a realização das tarefas e demais atividades cotidianas. A falta dessas habilidades tem sido considerada uma barreira à adoção do teletrabalho pelas organizações (Neirotti, Paolucci, \& Raguseo, 2012).

Os 10 itens da EHTC estão associados a uma escala de concordância Likert de 5 pontos, sendo 1 "Discordo totalmente" e 5 "Concordo totalmente". O instrumento foi submetido a processo de validação semântica junto aos profissionais de gestão de pessoas das organizações envolvidas. O questionário aplicado à amostra incluía também questões relativas a características sociodemográficas dos participantes (sexo, idade, nível de escolaridade, estado civil, se reside sozinho ou com outro(s) e informações sobre o contexto de trabalho - como organização, suporte tecnológico ao trabalho remoto e contexto de trabalho em casa).

\section{Procedimentos de Coleta de Dados e Cuidados Éticos}

A coleta de dados ocorreu de forma on-line, por meio da plataforma Survey Monkey, durante os meses de abril, maio e junho de 2020, nos quais os serviços considerados não essenciais eram realizados exclusivamente por meio remoto. Os links dos questionários, gerados pelos pesquisadores, foram encaminhados pelos dirigentes da área de gestão de pessoas aos servidores. No início do instrumento, constava o Termo de Consentimento Livre e Esclarecido (TCLE), que precisava ser aceito pelo respondente para que a coleta de dados pudesse ocorrer. O TCLE apresentava os objetivos da pesquisa e assegurava ao participante a liberdade de interromper a sua participação em qualquer fase da pesquisa, o sigilo das respostas individuais e o tratamento agrupado dos dados.

\section{Procedimentos de Análise de Dados}

Os dados coletados foram analisados com o apoio do software $\mathrm{R}$ ( $\mathrm{R}$ Core Team, 2018), com uso dos pacotes psych (Revelle, 2018), na fase exploratória; lavaan (Rosseel, 2012), na fase confirmatória; e qgraph (Epskamp, Cramer, Waldorp, Schmittmann, \& Borsboom, 2012), na análise de redes. Como complemento a essas evidências de validade da estrutura interna, foi também analisada a invariância (gênero e escolaridade) da EHTC.

A adequação dos dados aos pressupostos para Análise Fatorial Exploratória (AFE) foi avaliada por meio do Kaiser-Meyer-Olkin (KMO). Os critérios de retenção fatorial utilizados foram Kaiser-Gutman, Análise Paralela (Timmerman \& Lorenzo-Seva, 2011) e Análise de Redes (Golino \& Epskamp, 2017). A matriz de correlação estimada foi policórica, e método de rotação oblíquo (promax) foi 1000 iterações, indicados para fenômenos psicológicos (Damásio, 2012). A consistência interna foi analisada por meio do Alfa de Cronbach $(\alpha)$, sendo aceitáveis valores superiores a 0,70 (Hair, Anderson, Babin, \& Black, 2010).

A análise de redes foi utilizada na fase exploratória para confirmar a retenção de fatores e fornecer uma representação gráfica da estrutura e dinâmica das relações entre os itens (Golino \& Epskamp, 2017). Esse método tem a vantagem de extrair e estimar fatores controlando o super ajuste, com uma penalização que fixa em zero as correlações parciais de baixa magnitude, por meio do índice Extended Bayesian Information Criterion (EBIC). Como resultado, há um melhor tratamento da multicolinearidade e das correlações espúrias dos itens, viabilizando compreensões mais apuradas acerca dos fatores psicológicos da escala.

A partir desse processo de penalização das correlações, a matriz de correlações parciais padronizadas é obtida e representada por meio de uma rede. A associação entre os itens é observada pela aresta que os liga, sendo a espessura e a intensidade da cor indicativas da magnitude da correlação entre os itens (Epskamp et al., 2012). Em relação ao posicionamento, itens mais correlacionados ficam mais próximos na rede, enquanto itens menos correlacionados se apresentam em posições mais afastadas.

$\mathrm{Na}$ Análise Fatorial Confirmatória (AFC), para estimações mais precisas e menos enviesadas para variáveis ordinais, a matriz de correlações policóricas foi submetida ao método de estimação Unweighted Least Squares Mean and Variance-Adjusted (ULSMV) robusto (Li, 2014). Os índices de confiabilidade foram o Alfa de Cronbach e a Confiabilidade Composta (CC), ambas (>0,70) (Hair et al., 2010). Os critérios para ajuste dos modelos foram: Comparative Fit Index (CFI) (>0,90), Tucker Lewis Index (TLI) $(>0,90)$, Root Mean Square Error of Approximation (RMSEA) $(<0,08)$ e Standardized Root Mean Square Residual (SRMR) $(<0,08)$ (Brown, 2015).

A invariância do instrumento foi analisada comparando a medida por meio da técnica de Análise Fatorial Confirmatória Multi- 
grupo (AFCMG). Para a categoria gênero, a amostra foi dividida em homens $(n=3.095,40,7 \%)$ e mulheres $(n=4.508,59,3 \%)$. Para a categoria escolaridade, foram consideradas etapas escolares completas, assim divididas: ensino médio $(n=503,6,6 \%)$, graduação $(n=2.506,32,9 \%)$, especialização $(n=4.006,52,7 \%)$ e mestrado e doutorado $(n=593,7,8 \%)$. A partir da definição desses grupos, foram realizados testes de invariância configural (modelo irrestrito), métrica, escalar e residual, nessa ordem hierárquica. Os modelos foram avaliados com base no CFI $(>0,90)$, índices de ajuste TLI $(>0,90)$ e RMSEA $(<0,08)$, com o intervalo de confiança de $90 \%$ não excedendo 0,10 . A invariância da medida baseou-se nos valores de diferença de CFI entre os modelos ( $\Delta$ CFI $<0,01)$ (Putnick \& Bornstein, 2016). Com a avaliação dessas condições, é possível afirmar se a configuração e os parâmetros de um instrumento são equivalentes para grupos diferentes (Damásio, 2013).

Por fim, após a confirmação da estrutura bifatorial da EHTC por meio da AFC, buscamos investigar as evidências de validade discriminante entre os dois fatores da escala, por meio do teste de diferença do qui-quadrado $\left(\chi^{2}\right)$ proposto por Rönkkö e Cho (2020). O método realizado, no ambiente do R, com a utilização do pacote semTools (semTools Contributors, 2021), comparou um modelo restrito - cuja correlação entre as habilidades foi fixada em 1 - a um modelo irrestrito (sem fixação de cargas).

\section{Resultados}

O modelo com melhores propriedades psicométricas e consistência teórica foi encontrado por meio de técnicas exploratórias e confirmatórias. Na etapa exploratória, foram extraídos dois fatores por meio dos métodos Kaiser-Gutman e Análise de Redes. A matriz de correlação policórica dos itens atendeu aos pressupostos da AFE, pois o KMO foi de 0,89, e as cargas fatoriais foram superiores a 0,40 (Tabela 1). A AFE foi realizada com os 10 itens originais da escala, indicando, pelo método de Kaiser-Gutman, dois fatores e a permanência de oito itens, sendo excluídos dessa versão os itens: Aut e DIG.

Tabela 1

Estrutura Fatorial da EHTC

\begin{tabular}{|c|c|c|c|c|}
\hline Código & Item & Soft Skills & Hard Skills & Singularidade \\
\hline Aut & $\begin{array}{l}\text { 1. Sinto-me capaz de executar as minhas atividades remotamente sem depender de } \\
\text { orientações de outras pessoas. }\end{array}$ & 0,158 & 0,302 & 0,818 \\
\hline $\mathrm{Adm} T$ & $\begin{array}{l}\text { 2. Sinto-me capaz de administrar meu tempo, conciliando o trabalho remoto com as } \\
\text { tarefas domésticas. }\end{array}$ & 0,849 & $-0,09$ & 0,326 \\
\hline Int & 3. Sinto-me capaz de estabelecer intervalos de descanso durante o período de trabalho. & 0,735 & $-0,08$ & 0,505 \\
\hline PrInf & $\begin{array}{l}\text { 4. Sinto-me capaz de descrever eventuais problemas de informática ao solicitar suporte } \\
\text { de alguém que está fisicamente distante. }\end{array}$ & 0,202 & 0,466 & 0,563 \\
\hline $\mathrm{DiG}$ & $\begin{array}{l}\text { 5. Sinto-me capaz de dialogar com o meu gestor sobre dificuldades relacionadas à } \\
\text { execução do meu trabalho. }\end{array}$ & 0,321 & 0,364 & 0,600 \\
\hline DiF & $\begin{array}{l}\text { 6. Sinto-me capaz de dialogar com a minha família e/ou com outras pessoas sobre a } \\
\text { necessidade de respeitarem o período em que estou trabalhando remotamente (evitan- } \\
\text { do interrupções). }\end{array}$ & 0,690 & 0,04 & 0,460 \\
\hline $\mathrm{Pla}$ & $\begin{array}{l}\text { 7. Ao planejar o meu dia a dia de trabalho, organizo-me para cumprir as metas estabe- } \\
\text { lecidas pelo meu gestor. }\end{array}$ & 0,548 & 0,218 & 0,505 \\
\hline EvInt & $\begin{array}{l}\text { 8. Sou capaz de evitar interrupções de outras pessoas quando estou concentrado } \\
\text { realizando uma tarefa. }\end{array}$ & 0,750 & $-0,04$ & 0,446 \\
\hline HabR & $\begin{array}{l}\text { 9. Tenho habilidade para participar de reuniões por meio de recursos de videoconfe- } \\
\text { rência. }\end{array}$ & $-0,134$ & 0,823 & 0,464 \\
\hline HabRC & $\begin{array}{l}\text { 10. Utilizo com facilidade os recursos de comunicação em conversas com o meu } \\
\text { gestor e demais membros da minha equipe. }\end{array}$ & $-0,144$ & 0,873 & 0,258 \\
\hline
\end{tabular}

Nota. As cargas marcadas em negrito permaneceram na estrutura da escala.

O resultado da AFE indicou, portanto, dois fatores correlacionados, a saber: (i) Soft Skills composto por cinco itens (AdmT, Int, DiF, Pla e EvInt); e (ii) Hand Skills composto por três itens
(HabR, HabRC e PrInf). Em seguida, houve a exploração dos dados com o emprego da técnica de análise de redes em que os itens são representados por vértices na rede e as arestas representam a associação entre dois itens. Com base nessa técnica, a Figura 1 ilustra a estrutura bifatorial da EHTC e as relações dos oito itens da rede que representam os fatores soft skills e hard skills. A magnitude das correlações positivas entre os itens é representada pela espessura de arestas; quanto mais espessa e intensa a cor, mais forte a sua associação.

$\mathrm{Na}$ fase confirmatória, buscou-se investigar se o modelo bifatorial se ajusta à matriz de correlação policórica dos itens. Essa matriz foi submetida ao método de estimação ULSMV robusto, pois possibilita estimações mais precisas e menos enviesadas para indicadores categóricos de nível ordinal que não atendem a pressupostos de normalidade.

O primeiro modelo testado, com os oito itens provenientes da AFE, não gerou índices de ajuste aceitáveis (Brown, 2015), em termos do RMSEA (Tabela 2). Assim, foi feita a análise do Modification index (Mi), que indicou alta correlação entre os erros dos itens Int e AdmT $(M i=849)$. A análise desse par de itens apontou que eles tinham conteúdos semelhantes. Nesse sentido, foi testado um segundo modelo, retirando da escala o item Int, uma vez que o item Adm'T apresentou uma carga fatorial padronizada mais elevada. Esse modelo apresentou melhoria dos índices em relação ao modelo original, mas ainda não atendia aos parâmetros desejáveis para uma AFC. A partir de uma nova análise do Mi, foram incluídas as correlações entre dois pares de itens, a saber: (i) DiF e EvInt; e (ii) HabR e HabRC. A correlação entre tais itens faz sentido teórico, uma vez que o conteúdo dos itens é próximo e permitiu chegar a um excelente ajuste de modelo (Tabela 2).

Nessa versão final, os indicadores do ajuste do modelo foram favoráveis, confirmando que a estrutura bifatorial apontada na fase exploratória é robusta. No modelo final com sete itens, verificou-se que os itens compartilham razoável variância e formam uma estrutura subjacente de dois fatores com adequada consistência interna ( $\alpha=0,90$ e $\alpha=0,85$; CC $=0,90$ e CC $=0,82$, respectivamente, para soft skills e hard skills). 


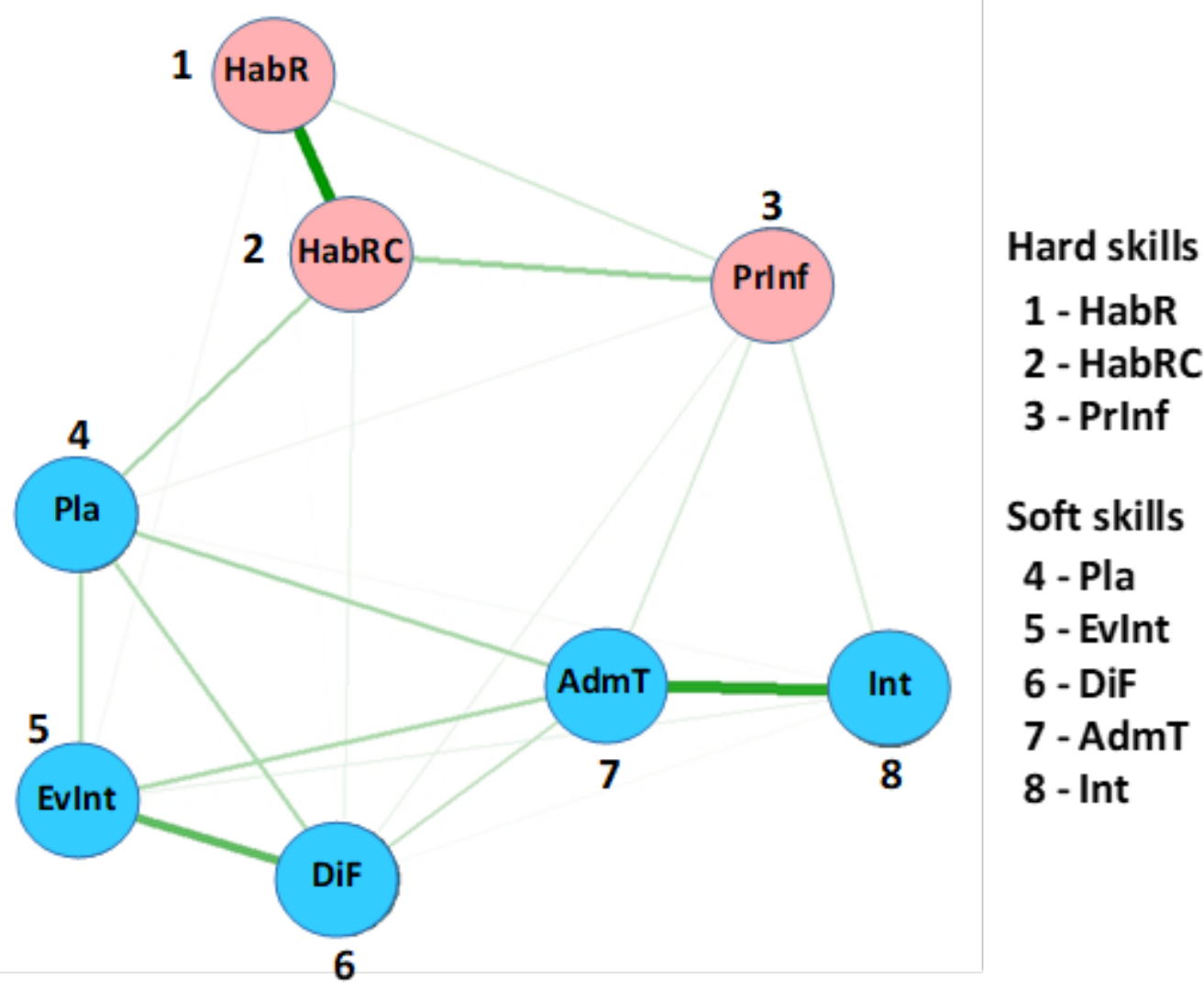

Figura 1. Rede da Escala de Habilidades para o Teletrabalho em Casa

Tabela 2

Análise fatorial confirmatória da EHTC

\begin{tabular}{|c|c|c|c|c|c|}
\hline Modelo & $\chi^{2}(g l)$ & CFI & TLI & RMSEA & SRMR \\
\hline $\begin{array}{c}\text { Modelo } 1 \text { - } \\
\text { Bifatorial (8 } \\
\text { itens) }\end{array}$ & $1044,39(19)$ & 0,969 & 0,955 & $\begin{array}{c}0,119 \\
(0,113-0,125)\end{array}$ & 0,043 \\
\hline $\begin{array}{c}\text { Modelo } 2 \text { - } \\
\text { Bifatorial ( } 7 \\
\quad \text { itens) }\end{array}$ & $567,94(13)$ & 0,981 & 0,969 & $\begin{array}{c}0,106 \\
(0,098-0,113)\end{array}$ & 0,035 \\
\hline $\begin{array}{c}\text { Modelo } 3 \\
\text { - Bifatorial } \\
\text { (7 itens) } \\
\text { Ajust. }\end{array}$ & $17,32(11)$ & 0,994 & 0,989 & $\begin{array}{c}0,062 \\
(0,054-0,070)\end{array}$ & 0,018 \\
\hline
\end{tabular}

Após a AFC, foi verificada a invariância da medida a depender do gênero ou do nível de escolaridade do trabalhador. A análise de invariância busca investigar o pressuposto de que a medida apresenta adequadas e semelhantes propriedades psicométricas para diferentes participantes (Damásio, 2013). Para tal, por meio de testes hierárquicos, a partir do modelo configural (irrestrito), foram sendo comparados modelos mais restritos - métrico, escalar e residual. A Tabela 3 apresenta os parâmetros da AFCMG. Foi verificada a invariância configural tanto para gênero quanto para nível de escolaridade. Desse modo, a estrutura bifatorial do instrumento é plausível para todos os grupos analisados, e o instrumento é invariante quanto às cargas fatoriais, aos interceptos e aos erros dos itens.

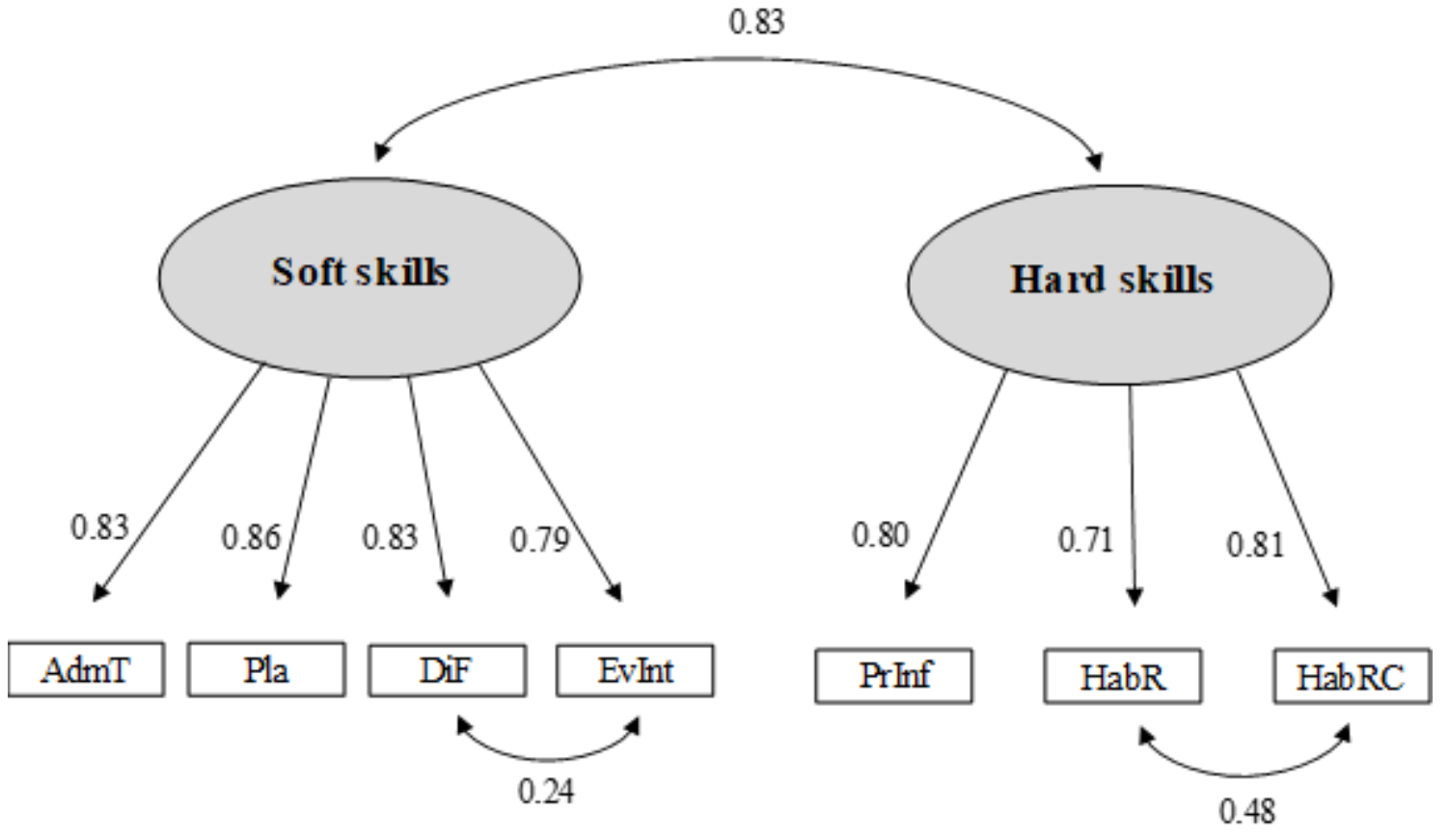


Tabela 3

Análise Fatorial Confirmatória Multigrupo da EHTC

\begin{tabular}{ccccc}
\hline $\begin{array}{c}\text { Invariância de } \\
\text { Gênero }\end{array}$ & RMSEA $(90 \%)$ & TLI & CFI & $\Delta$ CFI \\
\hline $\begin{array}{c}\text { Modelo Configural } \\
\text { (Irrestrito) }\end{array}$ & $0,06(0,05-0,07)$ & 0,99 & 0,99 & - \\
$\begin{array}{c}\text { Invariância } \\
\text { Métrica }\end{array}$ & $0,03(0,02-0,04)$ & 0,99 & 0,99 & 0,004 \\
$\begin{array}{c}\text { Invariância Escalar } \\
\text { Invariância } \\
\quad \text { Residual }\end{array}$ & $0,02(0,01-0,03)$ & 0,99 & 0,99 & 0,001 \\
$\begin{array}{c}\text { Invariância de } \\
\text { Escolaridade }\end{array}$ & $0,03(0,02-0,03)$ & 0,99 & 0,99 & 0,002 \\
$\begin{array}{c}\text { Modelo Configural } \\
\text { (Irrestrito) }\end{array}$ & $0,06(0,05-0,07)$ & 0,99 & 0,99 & - \\
$\quad \begin{array}{l}\text { Invariância } \\
\text { Métrica }\end{array}$ & $0,02(0,01-0,03)$ & 0,99 & 0,99 & 0,005 \\
$\begin{array}{c}\text { Invariância Escalar } \\
\text { Invariância } \\
\quad \text { Residual }\end{array}$ & $0,01(0,01-0,02)$ & 0,99 & 0,99 & 0,001 \\
\hline
\end{tabular}

Apesar da correlação de alta magnitude entre as soft e hard skills $(r=0,83 ; p<0,001)$, os resultados indicaram evidências de validade discriminante. $\mathrm{O}$ teste de diferença do qui-quadrado indicou que os fatores não são similares $\left(\Delta \chi^{2}[1]=70,21, p<0,001\right)$. Assim, apesar da esperada correlação entre os fatores, uma vez que pertencem a um mesmo construto, o fator de hard skills se distingue estatisticamente do fator de soft skills. Dessa forma, há argumentos teóricos e empíricos para considerar a estrutura interna da EHTC como bifatorial.

\section{Discussão}

O presente estudo pretendeu relatar o desenvolvimento e as evidências de validade psicométrica da Escala de Habilidades para o Teletrabalho em Casa (EHTC). Os itens da EHTC foram construídos tendo como base a literatura sobre o teletrabalho realizado em casa e o contexto de implantação mandatória dessa modalidade devido à pandemia de COVID-19. O objetivo foi cumprido ao propor uma medida que considera uma situação específica de trabalho remoto e apresentar sua estrutura fatorial, consistência interna e invariância.

A EHTC possui sete itens divididos em dois fatores: um fator para as soft skills e outro para as hard skills. Esses aspectos englobam, respectivamente, habilidades para o balanceamento entre trabalho e outras atividades e para a utilização de recursos digitais de comunicação nas interações de trabalho. Essa confirmação reveste-se de importância, pois o teletrabalho vem sendo adotado por diferentes setores e organizações, como é o caso do setor público brasileiro, e conhecer as diferentes habilidades dos servidores para essa modalidade pode contribuir para processos mais eficazes de implementação e de gestão do teletrabalho.

O primeiro fator da EHTC avalia soft skills, habilidades intrapessoais e interpessoais, consideradas recursos pessoais positivos e valiosos para o enfrentamento do aumento de demandas cognitivas e afetivas no trabalho (Charoensap-Kelly et al., 2015; Cinque, 2016). Entre essas soft skills mensuradas pela escala, estão a conciliação do trabalho com outros afazeres (tarefas domésticas, acompanhamento de filhos em ensino remoto etc.) e o estabelecimento de limites entre as fronteiras trabalho e não trabalho (lazer, descanso e vida pessoal). A busca do equilíbrio trabalho-família, além de habilidades de gestão do tempo e compatibilização de horários de trabalho e não trabalho, compreende a capacidade de evitar interferências e interrupções de outras pessoas durante os horários destinados ao teletrabalho (Kniffin et al., 2021; Wang et al., 2021).

Ruídos e interrupções de outras pessoas no teletrabalho feito a partir de casa, se não forem suprimidos, podem causar perda excessiva de energia do trabalhador e afetar o seu desempenho (Sandall \& Mourão, 2020; Windeler et al., 2017). Nos primeiros meses de combate à pandemia, muitos trabalhadores eram inexperientes na modalidade remota e não dispunham de um ambiente adequado (equipamentos, mobiliário, local isolado etc.) ao teletrabalho em casa, o que tornou ainda mais relevante a habilidade de dialogar com familiares para garantir um trabalho livre de interferências, distrações e ruídos.

O segundo fator da escala avalia hard skills e refere-se ao uso de tecnologias digitais para a comunicação síncrona e assíncrona com colegas e gestores, essenciais para o trabalho remoto feito integralmente em casa, que demanda a participação em reuniões virtuais e a comunicação mediada por tecnologias digitais (Sandall \& Mourão, 2020). A realização das tarefas em ambiente doméstico, ao ampliar a quantidade de atores (colegas, superiores e familiares) e aumentar bruscamente a variedade e a intensidade do uso de recursos de mediação tecnológica, demandou dos trabalhadores a aprendizagem imediata de bard skills para a comunicação síncrona e assíncrona com colegas e gestores. Assim, as características sociais do trabalho remoto durante a pandemia, ao demandar o uso intensivo de tecnologias digitais de mediação para a realização das tarefas, aumentaram as demandas de gestão de equipes virtuais e dificultaram a compatibilização de horários síncronos de trabalho e a comunicação instantânea e contingente entre colegas e gestores.

As hard skills, além de possibilitarem a realização do trabalho remoto em casa, podem ter facilitado ao teletrabalhador a busca e a oferta do suporte social necessário ao enfrentamento da perda dos contatos face a face, da redução das redes de apoio ao trabalho doméstico e do aumento de sentimentos de solidão e de isolamento social, ocorridos principalmente durante as medidas de controle da pandemia, como o distanciamento social e o trabalho remoto não voluntário em casa (Wang et al., 2021). Em parte, o relacionamento positivo entre as hard e soft skills pode ter ocorrido como um efeito direto do uso intensivo de tecnologias digitais para todos ou quase todos os tipos de comunicação de trabalho, compras, transações financeiras e relacionamentos pessoais. Essa realidade, em que todas as relações são mediadas por tecnologias digitais sem encontros face a face, feitas a partir de casa e em tempo integral, pode ter diminuído temporariamente os limites entre soft skills e hard skills.

Possuir habilidades variadas, que combinem soft e hard skills, pode funcionar como um recurso pessoal propulsor de estados psicológicos e resultados positivos, mitigando ou diminuindo efeitos negativos exercidos pelo teletrabalho (não voluntário) sobre vivências psicológicas, bem-estar, satisfação e desempenho no trabalho. A EHTC mensura soft e hard skills relevantes ao enfrentamento de situações de desequilíbrio entre demandas e recursos, como a que caracteriza o período de pandemia de COVID-19, em que há riscos de esgotamento dos recursos pessoais devido ao aumento da carga de trabalho e à falta de momentos de descanso e recuperação de energia em um período prolongado de crise (Hobfoll, 2001; Demerouti et al., 2001; Meseguer-de Pedro et al., 2021).

Além de explorar e confirmar a estrutura interna da EHTC, também foi testada a invariância da medida, com vistas a registrar a qualidade psicométrica do instrumento para teletrabalhadores de diferentes gêneros e níveis de escolaridade. Desse modo, cabe destacar que a amplitude desse recorte sociodemográfico, no contexto do trabalho, sugere a ampla aplicação do instrumento nas organizações brasileiras. 
A obtenção de bons índices de consistência interna e indicadores de ajuste para o modelo bifatorial permite a indicação da EHTC para mensurar a adaptação e o preparo, em termos de habilidades, para atuar no teletrabalho em casa. Como ferramenta de diagnóstico, a escala pode ser utilizada antes da implementação da modalidade remota de trabalho, com vistas a avaliar o domínio ou a percepção de déficit de habilidades e propor intervenções cabíveis, como o oferecimento de suporte, por meio de políticas e práticas de gestão, para a melhor conciliação trabalho-família, ou ações educacionais para suprir as lacunas identificadas.

A robustez das técnicas de análise de dados utilizadas, o tamanho amostral para a verificação de evidências de validade psicométrica e a proposição de uma medida sintética e parcimoniosa, possibilitando celeridade na sua aplicação e na interpretação de resultados, são alguns pontos fortes deste estudo. Contudo, algumas limitações devem ser apontadas, como, por exemplo, a não inclusão de testes de validade convergente, concorrente ou preditiva. Cabe destacar que a validade concorrente permanece como um desafio para a EHTC, instrumento inovador no contexto nacional, haja vista a dificuldade em se encontrar instrumentos similares ou escalas de mensuração de construtos reconhecidamente correlacionados.

Para estudos futuros, a validade preditiva pode ser analisada em pesquisas longitudinais e a convergente, em redes nomológicas com outros construtos. No entanto, esse manuscrito contribui para o início desse percurso metodológico de consolidação dos instrumentos de medida para o teletrabalho no Brasil. Para isso, foram utilizados rigorosos métodos com vistas a garantir a validade interna e discriminante dos fatores da escala. Pesquisas futuras poderão utilizar a EHTC em outras amostras e contextos (inclusive em teletrabalho voluntário, com pequenos ajustes), bem como investigar possíveis associações entre essas habilidades e outros indicadores, como produtividade, bem-estar e engajamento no trabalho.

A EHTC pode ser utilizada para identificar demandas de aprendizagem de hard e soft skills, planejar e realizar intervenções adequadas à natureza da habilidade a ser desenvolvida, por meio de programas de treinamento, desenvolvimento, educação e promoção de qualidade de vida no trabalho. Também pode ser adotada em estudos sobre o equilíbrio entre recursos pessoais ( hard e soft skills), outros recursos (suporte social, gerencial, organizacional e tecnológico), demandas de trabalho (metas pouco flexíveis, falta de intervalos para descanso, jornadas longas de trabalho, excesso de trabalho síncrono e interdependente e falta de suporte gerencial) e seus efeitos sobre estados psicológicos, bem-estar, satisfação e produtividade.

\section{Referências}

Abbad, G. S., Legentil, J., Damascena, M., Miranda, L., Feital, C., \& Neiva, E. (2019). Percepções de teletrabalhadores e trabalhadores presenciais sobre desenho do trabalho. Revista Psicologia Organizações e Trabalho, 19(4), 772-780. https://doi.org/10.17652/rpot/2019.4.17501

Abbad, G. S., \& Legentil, J. (2020). Novas demandas de aprendizagem dos trabalhadores face à pandemia da COVID-19. Em M. M. Moraes (Ed.), Os impactos da pandemia para o trabalhador e suas relações com o trabalho (pp. 45-58, Vol. 2, Coleção O trabalho e as medidas de contenção da COVID-19: Contribuições da Psicologia Organizacional e do Trabalho no contexto da pandemia). Porto Alegre: Artmed. Recuperado de https://www.sbpot.org. br/download/281

Agência Brasília (2020). GDF economiza R \$36 milhões com teletrabalho. Gastos com energia elétrica e água e esgoto foram os que mais tiveram queda nos prédios públicos durante a pandemia. Recuperado de https://www.agenciabrasilia.df.gov. br/2020/08/13/gdf-economiza-r-36-milhoes-com-teletrabalho/

Aguilera, A., Lethiais, V., Rallet, A., \& Proulhac, L. (2016). Home-based telework in France: Characteristics, barriers and perspectives. Transportation Research Part A: Policy and Practice, 92, 1-11. https://doi.org/10.1016/j.tra.2016.06.021
Bakker, A. B., \& Demerouti, E. (2017). Job demands-resources theory: Taking stock and looking forward. Journal of Occupational Health Psychology, 22(3), 273-285. https://doi.org/10.1037/ocp0000056

Barros, A. M., \& Silva, J. R. G. (2010). Percepções dos indivíduos sobre as consequências do teletrabalho na configuração home-office: Estudo de caso na Shell Brasil. Cadernos Ebape. BR, 8(1), 72-91. https://doi.org/10.1590/ S1679-39512010000100006

Biron, M., \& van Veldhoven, M. (2016). When control becomes a liability rather than an asset: Comparing home and office days among part-time teleworkers. Journal of Organizational Behavior, 37(8), 1317-1337. https://doi. org/10.1002/job.2106

Brasil (2021). Decreto $n^{\circ}$ 41.841: Dispõe sobre o teletrabalho, em caráter excepcional e provisório, para os órgãos da administração pública direta, indireta, autárquica e fundacional do Distrito Federal, como medida necessária à continuidade do funcionamento da administração pública distrital, em virtude da pandemia da COVID-19 e dá outras providências. Recuperado de http://www.sinj.df.gov.br/sinj/Norma/ f420758b53ef4ac698ae64733fc20726/Decreto $418412602 \quad 2021 . \mathrm{html}$

Brasil (2020). Instrução Normativa n ${ }^{\circ}$ 19: Estabelece orientações aos órgãos e entidades do Sistema de Pessoal Civil da Administração Pública Federal - SIPEC, quanto às medidas de proteção para enfrentamento da emergência de saúde pública de importancia internacional decorrente do coronavírus (COVID-19). Recuperado de https:// www.in.gov.br/en/web/dou/-/instrucao-normativa-n-19-de-12-de-marcode-2020-247802008

Brasil (2020). Instrução Normativa $n^{0}$ 65: Estabelece orientações, critérios e procedimentos gerais a serem observados pelos órgãos e entidades integrantes do Sistema de Pessoal Civil da Administração Federal-SIPEC relativos à implementação de Programa de Gestão. Recuperado de https://www.in.gov.br/en/web/dou/-/instrucaonormativa-n-65-de-30-de-julho-de-2020-269669395

Brown, T. A. (2015). Confirmatory factor analysis for applied research. Methodology in the social sciences $\left(2^{\mathrm{a}} \mathrm{ed}\right.$.). New York: The Guilford Press.

Charoensap-Kelly, P., Broussard, L., Lindsly, M., \& Troy, M. (2015). Evaluation of a Soft Skills Training Program. Business and Professional Communication Quarterly, 79(2), 154-179. https://doi.org/10.1177/2329490615602090

Cinque, M. (2016). "Lost in translation". Soft skills development in European countries. Tuning Journal for Higher Education, 3(2), 389-427. https://doi. org/10.18543/tihe-3(2)-2016pp389-427

Damásio, B. F. (2012). Uso da análise fatorial exploratória em psicologia. Avaliação Psicológica, 11(2), 213-228. Recuperado de http://pepsic.bvsalud.org/scielo. php? script $=$ sci abstract\&pid $=$ S1677-04712012000200007\&lng $=$ pt\&nrm $=$ iso\&tlng $=\mathrm{pt}$

Damásio, B. F. (2013). Contribuições da Análise Fatorial Confirmatória Multigrupo (AFCMG) na avaliação de invariância de instrumentos psicométricos. Psico-USF, 18(2), 211-220. https://doi.org/10.1590/S1413$\underline{82712013000200005}$

Demerouti, E., Bakker, A. B., Nachreiner, F., \& Schaufeli, W. B. (2001). The job demands resources model of burnout. Journal of Applied Psychology, 86(3), 499-512. https://doi.org/10.1037/0021-9010.86.3.499

Dingel, J. I., \& Neiman, B. (2020). How many jobs can be done at home? Journal of Public Economics, 189, 1-19. https://doi.org/10.3386/w26948

Epskamp, S., Cramer, A. O. J., Waldorp, L. J., Schmittmann, V. D., \& Borsboom, D. (2012). qgraph: Network visualizations of relationships in psychometric data. Journal of Statistical Software, 48(4), 1-18. https://doi.org/10.18637/jss. $\underline{\mathrm{v} 048.104}$

Eurofound (2015). European Working Conditions Surveys (EWCS). Recuperado de https://www.eurofound.europa.eu/pt/surveys/european-workingconditions-surveys/sixth-european-working-conditions-survey-2015

Goes, E. F., Ramos, D. O., \& Ferreira, A. J. F. (2020). Desigualdades raciais em saúde e a pandemia da Covid-19. Trabalho, Educação e Saúde, 18(3), e00278110. https://doi.org/10.1590/1981-7746-sol00278

Golino, H. F., \& Epskamp, S. (2017). Exploratory graph analysis: A new approach for estimating the number of dimensions in psychological research. PLOS ONE, 12(6), e0174035. https://doi.org/10.1371/journal.pone.0174035

Gondim, S. M. G., Morais, F. A., \& Brantes, C. A. A. (2014). Competências socioemocionais: Fator-chave no desenvolvimento de competências para o trabalho. Revista Psicologia, Organiz̧ações e Trabalho, 14(4), 394406. Recuperado de http://pepsic.bvsalud.org/scielo.php? script=sci arttext\&pid $=$ S1984-66572014000400006

Hair, J. F., Anderson, R. E., Babin, B. J., \& Black, W. C. (2010). Multivariate data analysis: A global perspective ( $7^{\mathrm{a}} \mathrm{ed}$.). New Jersey: Prentice Hall.

Hobfoll, S. E. (2001). The influence of culture, community, and the nested-self in the stress process: Advancing Conservation of Resources Theory. Applied Psychology, 50(3), 337-421. https://doi.org/10.1111/1464-0597.00062

Instituto Brasileiro de Geografia e Estatística (2018). Pesquisa Nacional por Amostra de Domicílios Continua - PNAD Contínua. Brasil. Recuperado de https:// www.ibge.gov.br/estatisticas/sociais/trabalho/9171-pesquisa-nacional-poramostra-de-domicilios-continua-mensal.html

Kniffin, K. M., Narayanan, J., Anseel, F., Antonakis, J., Ashford, S. P., Bakker, A. B., ... \& Vugt, M. V. (2021). COVID-19 and the workplace: Implications, issues, and insights for future research and action. American Psychologist, 76(1), 63-77. https://doi.org/10.1037/amp0000716 
Laker, D. R., \& Powell, J. L. (2011). The differences between hard and soft skills and their relative impact on training transfer. Human Resource Development Quarterly, 22(1), 111-122. https://doi.org/10.1002/hrdq.20063

Li, C. H. (2014). The performance of MLR, USLMV, and WLSMV estimation in structural regression models with ordinal variables (Doctoral dissertation). Michigan State University, East Lansing. https://doi.org/doi:10.25335/M58979

Losekann, R. G. C. B., \& Mourão, H. C. (2020). Desafios do teletrabalho na pandemia covid-19: Quando o home vira office. Caderno de Administração, 28(Ed. Esp.), 71-75. Recuperado de https://periodicos.uem.br/ojs/index. $\mathrm{php} / \mathrm{CadAdm} /$ article/view/53637

Luthans, F., Youssef, C., \& Avolio, B. (2007). Psychological capital: Investing and developing positive organizational behavior. Em D. L. Nelson, \& C. L. Cooper (Eds.), Positive Organizational Behavior (pp. 9-24). London: SAGE Publications Ltd. Recuperado de https://www.doi. $\operatorname{org} / 10.4135 / 9781446212752 . n 2$

Martins, L. B., Aguiar, C. V. N., \& Bastos, A. V. B. (2020). COVID-19: Seus impactos na relação trabalho-família. Em F. Queiroga (Ed.), Orientações para o bome office durante a pandemia da COVID-19 (pp. 49-58, Vol. 1, Coleção O trabalho e as medidas de contenção da COVID-19: Contribuições da Psicologia Organizacional e do Trabalho no contexto da pandemia). Porto Alegre: Artmed. Recuperado de https://www.sbpot.org.br/download/296

Meseguer-de Pedro, M., Soler-Sánchez, M. I., Fernández-Valera, M.-M., \& GarcíaIzquierdo, M. (2017). Evaluación del capital psicológico en trabajadores españoles: Diseño y estructura empírica del cuestionario OREA. Anales de Psicología, 33(3), 713-721. https://doi.org/10.6018/analesps.33.3.261741

Meseguer-de Pedro, M., Fernández-Valera, M. M., García-Izquierdo, M., \& SolerSánchez, M. I. (2021). Burnout, psychological capital and health during COVID-19 social isolation: A longitudinal analysis. International Journal of Environmental Research and Public Health, 18(3), 1064-1075. https://doi. org/10.3390/ ijerph18031064

Morgeson, F. P., \& Humphrey, S. E. (2006). Work Design Questionnaire (WDQ): Developing and validating a comprehensive measure for assessing job design and the nature of work. Journal of Applied Psychology, 91(6), 1321-1339. https://doi.org/10.1037/0021-9010.91.6.1321

Neirotti, P., Paolucci, E., \& Raguseo, E. (2012). Telework configurations and labour productivity: Some stylized facts. International Journal of Engineering Business Management, 4, 1-10. https://doi.org/10.5772/51641

Parker, S. K. (2014). Beyond motivation: Job and work design for development, health, ambidexterity, and more. Annual Review of Psychology, 65(1), 661-691. https://doi.org/10.1146/annurev-psych-010213-115208

Putnick, D. L., \& Bornstein, M. H. (2016). Measurement invariance conventions and reporting: The state of the art and future directions for psychological research. Developmental Review, 41, 71-90. https://doi.org/10.1016/j. dr.2016.06.004

R Core Team (2018). R: A language and environment for statistical computing. R Foundation for Statistical Computing. Recuperado de http://www.R-project.org/

Raghuram, S., Hill, N. S., Gibbs, J. L., \& Maruping, L. M. (2019). Virtual work: Bridging research clusters. Academy of Management Annals, 13(1), 308-341. https://doi.org/10.5465/annals.2017.0020

Revelle, W. (2018) Psych: Procedures for personality and psychological research. Northwestern University. Recuperado de https://CRAN.R-project.org/ package $=$ psych Version $=1.8 .4$.

Rönkkö, M., \& Cho, E. (2020). An updated guideline for assessing discriminant validity. Organizational Research Methods, 1-42. https://doi. org/10.1177/1094428120968614

Rosseel, Y. (2012). lavaan: An R package for structural equation modeling and more, Version 0.5-12 (BETA). Journal of Statistical Software, 48(2), 1-36. https://doi.org/10.18637/jss.v048.i02

Sandall, H., \& Mourão, L. (2020). Desempenho no trabalho: Desafios para trabalhadores e gestores em teletrabalho compulsório. Em F. Queiroga (Ed.), Orientações para o home office durante a pandemia da COVID-19 (pp. 21 30, Vol. 1, Coleção O trabalho e as medidas de contenção da COVID-19: Contribuições da Psicologia Organizacional e do Trabalho no contexto da pandemia). Porto Alegre: Artmed. Recuperado de https://www.sbpot.org. br/download/296

sem'Tools Contributors (2021). semTools: Useful tools for structural equation modeling. $\mathrm{R}$ package version 0.5-4. Recuperado de https://CRAN.R-project.org/ package $=$ semTools

Timmerman, M. E., \& Lorenzo-Seva, U. (2011). Dimensionality assessment of ordered polytomous items with parallel analysis. Psychological Methods, 16(2), 209-220. https://doi.org/10.1037/a0023353

Wang, B., Liu, Y., Qian, J., \& Parker, S. K. (2021). Achieving effective remote working during the COVID-19 pandemic: A work design perspective. Applied Psychology, 70(1), 16-59. https://doi.org/10.1111/apps.12290

Windeler, J. B., Chudoba, K. M., \& Sundrup, R. Z. (2017). Getting away from them all: Managing exhaustion from social interaction with telework. Journal of Organizational Behavior, 38(7), 977-995. https://doi.org/10.1002/job.2176
Informações sobre os autores:

\section{Gardênia da Silva Abbad}

E-mail: gardenia@unb.br

Luciana Mourão

E-mail: mourao.luciana@gmail.com

Russencleyton Barros Costa

E-mail: russencleyton@gmail.com

Lara Barros Martins

E-mail: 1barros@uloyola.es

\section{Juliana Legentil}

E-mail: julianalegentil@gmail.com

Lisa Miranda

E-mail: lisa.fdmiranda@gmail.com 